\title{
A Containerized Approach for Allocating Distributed Stream Queries to Fog Nodes
}

\author{
Hamed Hasibi, Saeed Sedighian Kashi
}

\begin{abstract}
Fog computing brings cloud capabilities closer to the Internet of Things (IoT) devices. IoT devices generate a tremendous amount of stream data towards the cloud via hierarchical fog nodes. To process data streams, many Stream Processing Engines (SPEs) have been developed. Without the fog layer, the stream query processing executes on the cloud, which forwards much traffic toward the cloud. When a hierarchical fog layer is available, a complex query can be divided into simple queries to run on fog nodes by using distributed stream processing. In this paper, we propose an approach to assign stream queries to fog nodes using container technology. We name this approach Stream Queries Placement in Fog (SQPF). Our goal is to minimize endto-end delay to achieve a better quality of service. At first, in the emulation step, we make docker container instances from SPEs and evaluate their processing delay and throughput under different resource configurations and queries with varying input rates. Then in the placement step, we assign queries among fog nodes by using a genetic algorithm. The practical approach used in SQPF achieves a near-the-best assignment based on the lowest application deadline in real scenarios, and evaluation results are evidence of this goal.
\end{abstract}

Index Terms-Assignment Problem, Docker, Genetic Algorithm, Stream Processing

\section{INTRODUCTION}

W ITH the rapid development of the Internet and Information technology, the speed of information production significantly increased [1], so the concept of big data introduced to analyze large amounts of information with volume, velocity, and variety characteristics [2]. There are general processing models for analysis and computation, such as batch processing and stream processing with big data. Usually, massive data-historical structures such as Wikipedia information or medical data contain high volumes and high data diversity. This type of data stores in distributed file systems due to its high volume and processes by batch processing systems. Conversely, stream processing systems are profitable for highspeed production and high diversification data, such as IoT sensors, financial transactions, and website clicks [3].

Batch processing concepts, solutions, and technologies such as MapReduce, Hive, and Pig are well developed and widely using in the industry. They have successfully solved the problem of big data processing [4]. However, for batch processing, at first, it needs to store large amounts of information in a database or file system. After spending a great deal of time gathering information, more consuming time is required to complete the analytical work on batch processing. The goal of batch processing is high throughput, not delay [5].

Hamed Hasibi and Saeed Sedighian Kashi are with the Faculty of Computer Engineering, K. N. Toosi University of Technology, Tehran, Iran (E-mails: Hamed.Hasibi@email.kntu.ac.ir, Sedighian@kntu.ac.ir).

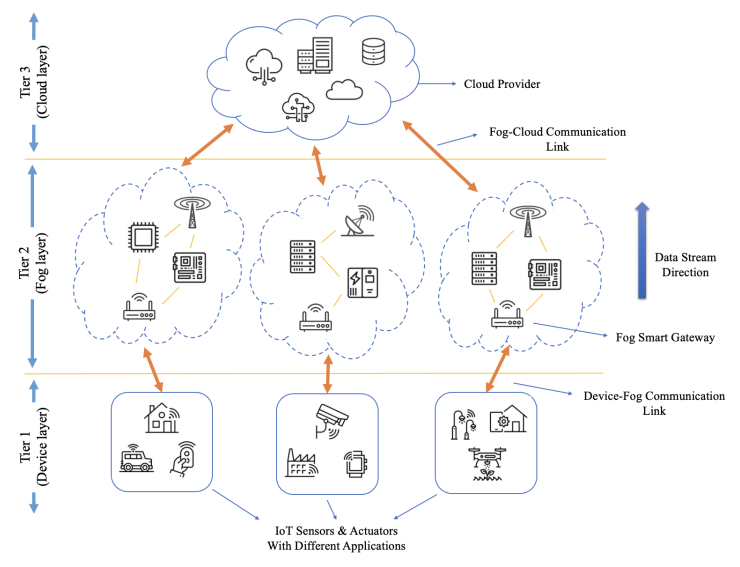

Fig. 1. The fog-cloud data stream architecture

Cloud facilities are not suitable for real-time processing demands of IoT applications. Therefore, some data processing is delegated to nodes close to the devices at the edge, in the fog layer, to allow data analysis with the least network latency [6]. The data must be processed while crossing the fog nodes before reaching the cloud for timely processing. The fog layer, unlike the cloud, has limited hardware resources and cannot store whole data. Therefore, besides the batch processing of data in the cloud nodes, it was necessary to invent transient data stream processing at fog nodes. To meet the growing demand for stream processing, stream engines such as Apache Storm, Apache Spark, Apache Flink, IBM Infosphere, and WSO2 stream processors have been designed [6].

General fog-cloud architecture is shown in Fig. 1. Tier 1 consists of end-devices such as sensors and cell phones that send data to tier 2, the fog layer, via a smart fog gateway. The smart fog gateway is responsible for receiving data from the first layer and dividing queries between fog nodes by establishing the fog nodes' routing. In this structure, the enddevices' data move from tier 1 to tier 3, the cloud layer. Usually, this data transition has been modeled with Directed Acyclic Graph (DAG) data structure [7]. The direction of this graph is from the bottom tier to the top tier. After partial processing of the data in tier 2 to meet the applications' requirements, such as minimum delay, the data will be sent to tier 3, where massive statistical processing is performed in the cloud.

Ignoring partial processing by tier 2 leads to considerable network delay, application deadline failure, and increased data processing workload in tier 3. Generally, by moving from the bottom to top layers, the hardware resources (processing, 

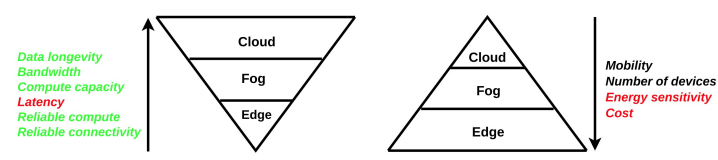

Fig. 2. Cloud and fog characteristics [8]

memory, cache, bandwidth capacity) increases. As a negative effect, the network link delay rises, too. Fig. 2 illustrates the mentioned fact.

Each application, such as fraud and unexpected event detection and camera surveillance systems, have their specific queries and sensors. For example, in addition to filter and aggregation queries, camera surveillance systems have their dedicated stream queries, such as convolutions and logical operators. Each application uses various sensors and has queries with different parameters. The stream processing queries placement on fog nodes affects QoS metrics such as latency, throughput, hardware usage, and energy consumption.

\section{A. Problem definition}

In real-world scenarios, there are several sensors and fog nodes with several applications. Each application has its stream queries and dedicated deadlines. Application queries consist of a set of queries in sequence, one after another. We aim to process the sensors' stream data on the fog nodes to meet end-to-end applications' deadlines. In other words, our goal is the placement of the queries on the fog nodes to minimize the end-to-end delay. The end-to-end delay consists of processing delay on nodes and transmission delay among nodes, from receiving data by smart fog node to reaching data to the cloud.

\section{B. Solution in brief}

Our approach, named Stream Queries Placement in Fog (SQPF), has two main steps. In the first step, using emulation, we have examined Stream Processing Engines (SPEs) using single-node Docker containers to find real-world performance metrics. Afterward, we set up a simulation assuming multinode fog architecture with the help of results obtained from the emulation phase. In other words, the steps are:

1) Emulation step: Calculating processing delay and throughput for single-node deployment. Processing delay is the time difference between the data arriving at the SPE input port and approaching processed data at its output port. Throughput is the number of outputs per unit of time [9] for each defined query using different deployed SPEs, resource configurations, and input stream rates.

2) Simulation step: Computing the best possible query placement on fog nodes using a Genetic Algorithm (GA). We use the results of emulation in the GA.

Our contributions are as follows:

- Introducing the emulation phase by using docker testbed to make any SPE performance evaluation possible. SQPF is a practical approach that uses real SPEs compared to many related works that simulate without using real SPEs.
This contribution is mentioned in the future works of a recent valuable survey on the topic [10].

- Proposing a new workflow consisting of nine subproblems to find a placement that minimizes end-to-end application delay.

- Introducing multi-SPE architecture on the fog layer.

The rest of the paper is organized as follows: In Section II, we describe related work. In Section III, SQPF steps are explained. In Section IV, we bring analysis and experiments about our solution. Finally, in Section V, we present the conclusion and future works.

\section{RELATED WORK}

Peng et al. [11] have presented R-Storm (Resource-Aware Storm), a system that implements resource-aware scheduling within Apache Storm components without considering fog characteristics and delay requirements. Pietzuch et al. [12] have introduced a stream-based overlay network (SBON), a layer between a stream-processing system and the physical network that manages operator placement for stream processing systems.

Amarasinghe et al. [13] devised an optimization framework consisting of a constraint satisfaction formulation and a system model that aims to minimize end-to-end latency through the appropriate placement of Data Stream Processing (DSP) either on cloud nodes or edge devices. Their evaluation schema is on a single server and doesn't represent multi-server fog architecture. Cardellini et al. [14] have studied the placement problem for distributed DSP applications. Their contributions are twofold. They have provided a general formulation of the optimal DSP placement as an Integer Linear Programming (ILP) problem that considers computing and networking resources' heterogeneity. They have presented a scheduler for the Apache Storm DSP framework. Our approach considers multiple SPEs and is not limited to a specific one.

Dias de Assunção et al. [15] have surveyed trending challenges and solutions proposed in distributed data stream processing and edge computing. Liu et al. [10] reviewed scheduling challenges in DSP systems and mentioned "integration of different SPEs" and "container-based deployment" as two significant gaps in the stream processing context. In this paper, we try to cover these two gaps. Cardellini et al. [16] have mentioned some relevant examples of recent research achievements defining new DSP applications in the fog computing context, such as run-time systems mechanisms and techniques targeting DPS frameworks, and also high-level interfaces for data streaming in highly dynamic IT environments. Yang [7] presented the general models and architectures of fog data streaming by analyzing the common properties of several typical applications. They also examined the design space of fog streaming with the consideration of four essential dimensions that are system, data, human, and optimization. Rodrigo De Souza et al. [17] have formulated the scenario of operator placement and parallelism as an optimal mixedILP. The proposed model is named Cloud-Edge data Stream Placement (CESP). Vanneste et al. [18] have presented the Distributed Uniform Stream (DUST) framework that creates 
an abstraction between the application components and the middleware. Next to the DUST framework, they also described the distributed DUST coordinator, which will optimize the resource consumption by moving the application components to different execution locations like fog nodes. Nardelli et al. [19] presented several DSP placement heuristics that consider computing and network resource heterogeneity.

Gosh et al. [20] have formulated an optimization problem for energy-aware placement of queries, composed as an analytics dataflow, across a collection of edge and cloud resources to minimize the end-to-end latency for the dataflow. Their evaluation is limited to the WSO2 stream processor and two resource configurations. Brogi et al. [21] have presented a container-based software architecture for supporting autonomic data stream processing applications over the fog computing network. Hiessl et al. [22] have designed a stream processing solution for the fog. They first have formulated an optimization problem for stream processing operators' placement, which is tailored to fog computing environments. Then, they build a plugin for stream processing frameworks that solves the optimization problem periodically to support the fog's dynamic resources. They evaluate this approach by performing experiments on an OpenStack testbed. By contrast, we use the docker container technology to dynamically scale up and down the fog nodes' resources assigned to the application components.

The summary of the comparison between our work and other closely related works is given in Table I.

TABLE I

RELATED WORK COMPARISON

\begin{tabular}{|l|c|c|c|c|c|c|c|c|c|c|c|c|}
\hline \multicolumn{1}{|c|}{ Parameter } & \multicolumn{6}{|c|}{ Related Work } & SQPF \\
\cline { 2 - 7 } & {$[11]$} & {$[12]$} & {$[13]$} & {$[14]$} & {$[17]$} & {$[18]$} & {$[19]$} & {$[20]$} & {$[21]$} & {$[22]$} \\
Pramework & $\checkmark$ & $\checkmark$ & $\checkmark$ & $\checkmark$ & $\checkmark$ & $\checkmark$ & $x$ & $x$ & $x$ & $x$ & $\checkmark$ \\
\hline $\begin{array}{l}\text { Bencharation } \\
\text { Tools }\end{array}$ & $\checkmark$ & $\checkmark$ & $\checkmark$ & $\checkmark$ & $x$ & $x$ & $\checkmark$ & $\checkmark$ & $\checkmark$ & $\checkmark$ & $\checkmark$ \\
\hline $\begin{array}{l}\text { Deadline } \\
\text { Consideration }\end{array}$ & $x$ & $\checkmark$ & $\checkmark$ & $x$ & $x$ & $x$ & $x$ & $\checkmark$ & $x$ & $x$ & $\checkmark$ \\
\hline $\begin{array}{l}\text { Containerizartion } \\
\text { Approach }\end{array}$ & $x$ & $x$ & $x$ & $x$ & $x$ & $x$ & $x$ & $x$ & $\checkmark$ & $x$ & $\checkmark$ \\
\hline $\begin{array}{l}\text { Multi-SPE } \\
\text { Mode }\end{array}$ & $x$ & $x$ & $x$ & $x$ & $x$ & $x$ & $x$ & $x$ & $x$ & $x$ & $\checkmark$ \\
\hline $\begin{array}{l}\text { Multi-node } \\
\text { Architecture }\end{array}$ & $\checkmark$ & $\checkmark$ & $\checkmark$ & $\checkmark$ & $\checkmark$ & $x$ & $\checkmark$ & $\checkmark$ & $\checkmark$ & $\checkmark$ & $\checkmark$ \\
\hline
\end{tabular}

\section{PROPOSED SQPF APPROACH}

There are a set of fog nodes with hierarchy architecture that receive data from IoT devices. Fog nodes can potentially receive input streams and send the output stream to the next fog node in order. This model of distributed stream processing is modeled by Eidenbenz et al. [23]. This system has its delay because of a) processing of some tasks by fog nodes, and b) sending data among nodes. The objective is to minimize the streaming delay for all tasks by the hierarchical fog nodes. The problem is NP-Hard [14][24].

The steps of SQPF is demonstrated in Fig. 3. In emulation phase, we evaluate SPE performance in standalone mode to find processing delay and throughput of SPEs under resource configurations and query conditions with varying rates of input. In simulation phase, we calculate the end-to-end delay for each application. An application has multiple queries in the hierarchical fog layer. By using processing delays achieved from emulation step and the network link (transmission) latency among fog nodes, we make fitness function for our GA solution. $(R)$ : $(C P U, R A M)$ indicate fog processor cores and memory in megabytes. $(Q)$ refers to a specific query in an application query sequence and (I) to SPE model like Apache Flink or Apache Storm.

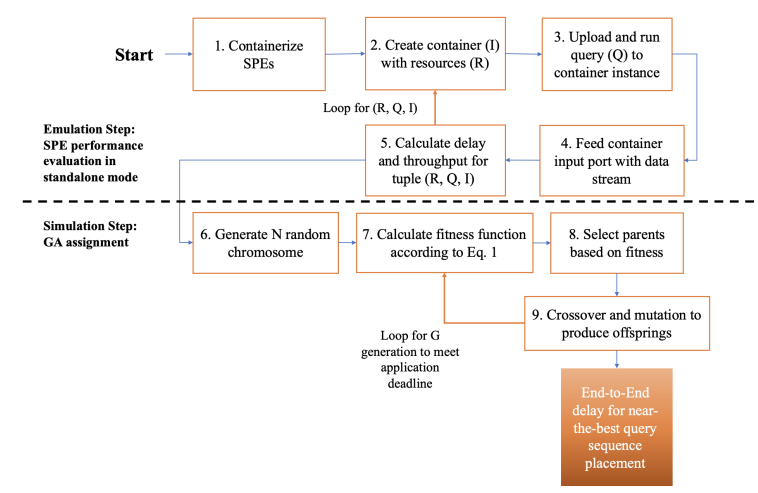

Fig. 3. SQPF steps

\section{A. Architecture}

Fig. 4 shows our proposed SQPF architecture. This structure consists of a workload pool, image container pool, benchmark tools, and the GA scheduler. Image container pool is a docker image repository consisting of all SPEs used in fog layers, including Apache Storm, Apache Flink, etc. For each SPE, there are related queries in the workload pool. For example, there are two query versions for the filter query corresponding to Apache Flink and WSO2 stream processor, respectively, in (.jar) and (.siddhi) file format. Benchmark tool calculates processing delay and throughput via Apache JMeter data generator at defined input rate to SPE container port. As noted before, it has been proved that scheduling stream processing task among nodes is an NP-hard problem [14][24]. Hence, we have used a heuristic algorithm to determine the near-the-best query sequence placement on the fog layer to minimize the end-to-end delay.

\section{B. SQPF details}

As we mentioned before, there is two major steps to find the near-the-best query sequence placement. In this section, we explain each step's details, according to Fig. 3 as follows:

1) Containerizing SPEs: First, we create an image from each SPE. It may need to gather engine files and write DockerFile to create an image. The following items are the benefits of using docker in our solution:

- All SPEs can be containerized. These docker images provide an integrated benchmarking solution to determine QoS metrics, including processing delay and throughput 


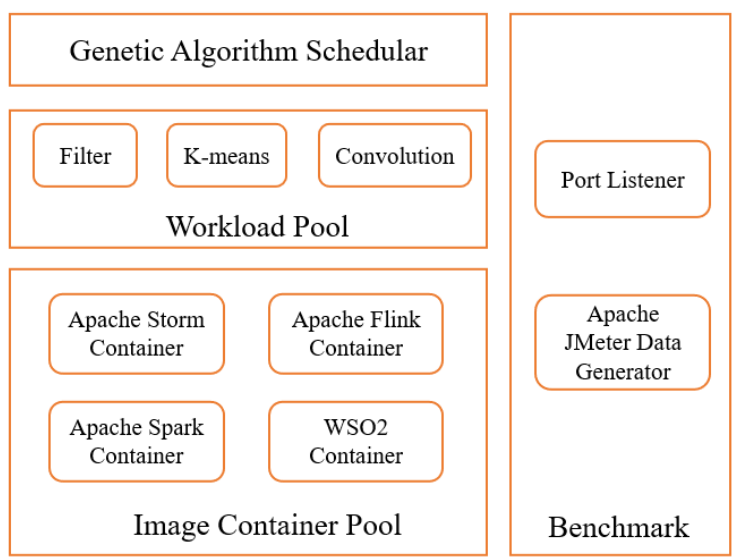

Fig. 4. SQPF architecture

for all engines. The engine programming language compatibility, for example, Java for Apache Flink, doesn't affect the benchmarking solution.

- Docker can expose a port to receive and send data streams. This feature will be used to send and receive high-rate streaming data at input/output ports. We use Apache JMeter to produce the simulation stream.

- Using docker lets multiple queries to be hosted on a single fog node resource without interference. This type of virtualization is a crucial capability for optimal resource allocation. The node hardware resources can be divided by a certain ratio between several queries [25].

2) Creating the container with specified resources: Here, we run containers with fog node resource characteristics (RAM and CPU) to emulate a run time environment under a particular SPE and query conditions:

docker run -it $-d-p$ [Ports] -memory $=[R A M$ amount of fog node $]-$ cpus $=$ [Number of fog node processing cores] -name [Container name] [Image name]

3) Upload and run query (Q) to container instance: At this step, we upload an appropriate query (i.e., filter or average query) from the workload pool to run at the container instance.

4) Feed container input port with a stream: After uploading and running the query, we feed the container's input port with generated stream data to stress the SPE container instance. We have used Apache JMeter to simulate data streams. Apache JMeter is an Apache project that can be used as a load testing tool for analyzing and measuring various services' performance. This tool allows generating multi-thread requests toward the engine container [26]. All inputs and outputs in queries are declared as HTTP TCP protocol.

5) Calculating processing delay and throughput for $(R$, $Q$, I) tuple: The processing delay and throughput on the container instance changes by varying the input rates. To obtain the processing delay, we use the timestamp in each data stream. After processing, when data reaches the output port, we compare the data timestamp to the machine time to obtain the processing delay. The output port has monitored to determine the number of output data per unit of time as a throughput value. Afterward, the output port bandwidth has measured with network monitoring software to obtain throughput in megabytes or gigabytes per unit of time. In a fog layer consisting of $R$ different node resource configurations, $I$ various SPEs, and $Q$ different query types in a specific application query sequence, we examine $R * Q * I$ states to calculate processing delay and throughput.

6) Generating $N$ random chromosomes: We use GA for placement as a heuristic approach. We enumerate fog nodes with the Breadth-first search (BFS) algorithm from bottom to top layers of fog. Fig. 5 demonstrates an application query sequence with three queries and a fog network with nine nodes. In Fig. 5, query numbers 1, 2, and 3 are dedicated to node numbers 1, 2, and 9, respectively. In Equation 1, $A\left(Q_{i}\right)$ is the assignment of a query $(i)$ to a node.

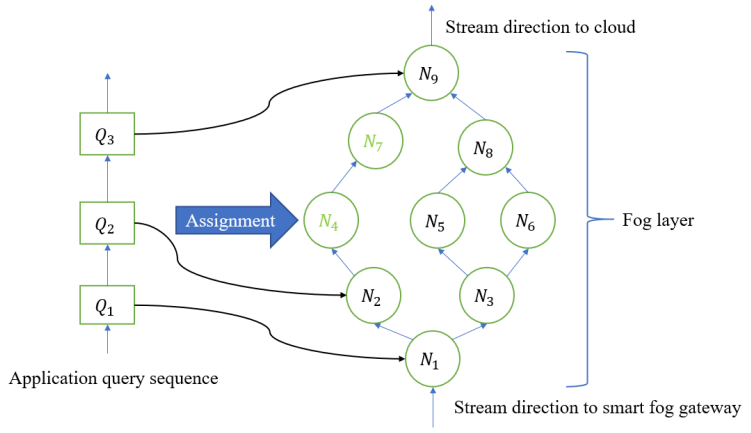

Fig. 5. Placement of three queries of an application in a fog network with nine nodes

$$
A\left(Q_{i}\right)=\left[\text { Node }_{i}, \text { Image }_{m}, C P U_{n}, R_{A} M_{p}\right]
$$

In the mentioned example, $A\left(Q_{1}\right)=[3,1,2,64]$ defines that query number 1 has been dedicated to node number 3 with Apache Flink engine using two processor cores and 64 megabytes of RAM.

In this scheduling problem, we have defined three assignment chromosomes. In chromosome type 1, the gene index refers to the query number and the gene value to the fog node number. For example, the first chromosome in Fig. 6 indicates that queries numbered 1,2 , and 3 are assigned to nodes numbered 1,1 , and 2 , respectively.

$$
\text { Population:- } \begin{array}{c|c|c|c|}
Q_{1} & Q_{2} & Q_{3} \\
\hline 1 & 1 & 2 \\
\hline 2 & 4 & 5 \\
\hline 3 & 9 & 3 \\
\hline \multicolumn{3}{|c|}{\vdots} \\
\hline 1 & \vdots & 6 & 7 \\
\hline
\end{array}
$$

Fig. 6. Chromosome type 1 schema

The number of cores and the amount of RAM for each assignment is added to chromosome type 1 genes to create chromosome type 2. For example, the first chromosome in Fig. 7 indicates that query 1 is assigned to node 1 with 1.5 CPU cores and 128MB of RAM.

In chromosome type 3, chromosome type 2 genes have been expanded to specify the used SPE. For this chromosome 


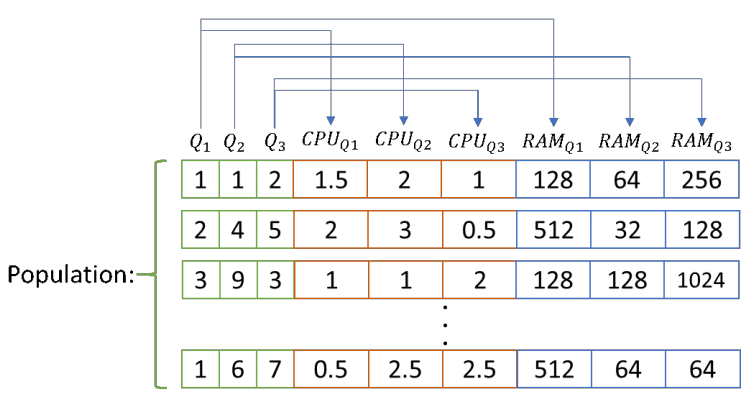

Fig. 7. Chromosome type 2 schema

type, the output data stream structure from an SPE should be as same as the input data stream structure at its next corresponding SPE (like JSON format) to orchestrate correctly. For example, the first chromosome in Fig. 8 indicates that query number $2\left(Q_{2}\right)$ assigned on node number 1 , executing on the SPE type 1 (Apache Flink) while its next corresponding query $\left(Q_{3}\right)$ has been assigned to node number 2 with SPE type 2 (Apache Storm).

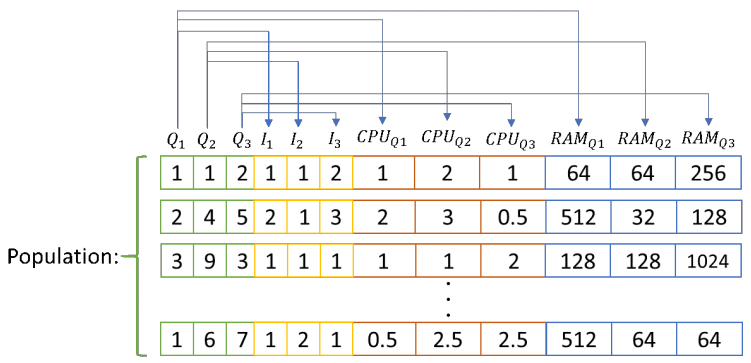

Fig. 8. Chromosome type 3 schema

7) Calculating fitness function for chromosomes: After choosing chromosome type, we define GA's fitness, Equation 2 , based on processing delays achieved from the simulation step. Link latencies and link transmition delay has been considered in formulation.

$$
\begin{array}{r}
\text { Fitness }=\sum \text { Delay }_{\text {processing }}+\sum \text { Latency }_{\text {Link }} \\
+\sum \frac{\text { EventSize }_{\text {Bandwidth }}}{\text { Link }}
\end{array}
$$

8) Selecting parents based on the fitness function: In this step, chromosomes will be chosen according to their fitness. Chromosomes with smaller fitness have a lower end-to-end delay and better performance.

9) Crossover and mutation to produce offspring: Chromosome types 1, 2, and 3 have one, three, and four separate parts, respectively. Chromosome type 3 has node, engine, $\mathrm{CPU}$, and RAM parts. Each part has its own crossover and mutation separately. For crossover operation, offspring takes half of its genes from one parent and the other half from its next parent. For mutation operation, a random gene from the parent chromosome changes to place the query on another node. After some generations, the end-to-end delay for the application query sequence improves and reaches near to its defined deadline.

\section{Performance Evaluation}

In this section, we first provide the results related to the emulation step in subsections IV-A and IV-B. Then the outputs for the simulation step will be shown in subsection IV-C.

In the subsection IV-A, we have some experiments on the problem variables that are SPE types, resource configurations, and query types. Also, the results of subsection IV-A exclude invalid chromosomes from offspring and speed up GA allocation approach. These experiments are very beneficial for the allocations involved in hundreds of queries and thousands of fog nodes.

In subsection IV-B, we inspect container instance usage under query stress to find out RAM and CPU utilization percentage.

In subsection IV-C, we have examined our solution on different fog layers with different application query sequences. Table II shows the hardware configuration, SPEs, and GA parameters used for this section experiments.

TABLE II

HARDWARE CONFIGURATION, SPES SPECIFICATIONS, AND GA PARAMETERS

\begin{tabular}{|l|c|}
\hline \multicolumn{2}{|c|}{ Default Parameters } \\
\hline \hline Apache Flink & Version: 4.3 .0 \\
WSO2 Stream Processor & Version: 1.8 .3 \\
RAM & 2 GB LPDDR3 2133 MHz \\
CPU & Intel 8259 U 2.3 GHz (2 out of 4 cores used) \\
Operating system & Ubuntu 18.04.2 LTS \\
Crossover Probability & $30 \%$ \\
Mutation Probability & $10 \%$ \\
\hline
\end{tabular}

\section{A. Analysis on problem variables}

1) Analysis of SPEs: Fig. 9 demonstrates WSO2 and Apache Flink comparison on latency and performance for filter queries with varying input rates. Using these comparison results, we can exclude several chromosomes from offspring. The mentioned experiment demonstrates the allocation $A\left(Q_{\text {filter }}\right)=[\mathrm{y}, 2,2,2]$ is better than the allocation of $A\left(Q_{\text {filter }}\right)=[\mathrm{y}, 1,2,2]$. As a result, all chromosomes with $A\left(Q_{\text {filter }}\right)=[\mathrm{y}, 1,2,2]$ will be excluded from offspring.

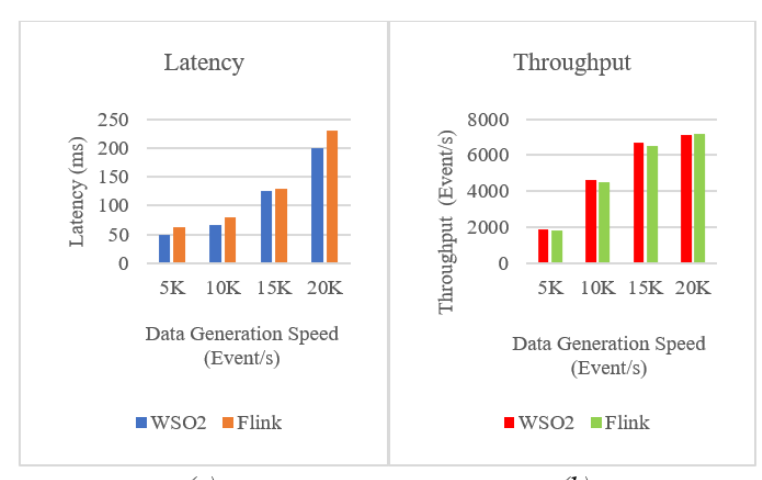

(a)

(b)

Fig. 9. Comparison between WSO2 and Apache Flink engines for filter query on a) Latency and b) Throughput 
2) Analysis of hardware resource configurations: The resource configuration effect on delay and throughput can be analyzed on a specific SPE with a particular query. In Fig. 10 , the impact of memory used for WSO2 SPE in filter query with different input rates is shown. Using this experiment's results, we can estimate the delay and throughput of some assignments without an exact calculation. For the experiment mentioned in Fig. 10, the delay and throughput values of $A\left(Q_{\text {filter }}\right)=[\mathrm{x}, 2,2,2]$ and $A\left(Q_{\text {filter }}\right)=[\mathrm{y}, 2,2,4]$ assignments have been calculated. Therefore, we can estimate the delay and throughput value of $A\left(Q_{\text {filter }}\right)=[\mathrm{z}, 2,2,8]$. It is noticeable that this estimate is not linear and does not double by doubling RAM's amount.

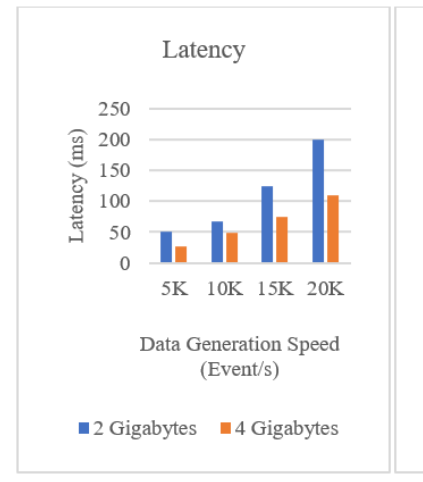

(a)

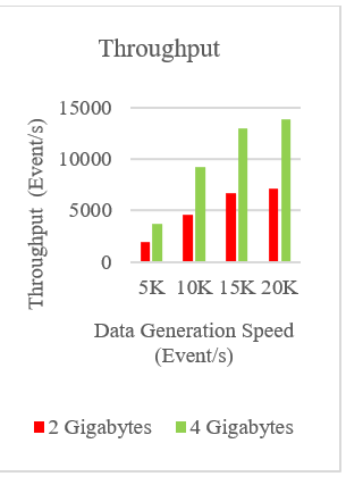

(b)
Fig. 10. The filter query a) delay and b) throughput comparison using WSO2 stream processor between two resource configurations: 2 GB 2133 MHz LPDDR3 memory and 4 GB $2133 \mathrm{MHz}$ LPDDR3 memory

3) Analysis of queries: Fig. 11 shows the effect of query on latency and throughput in WSO2 SPE. The analysis of processing delay and throughput of different queries reveals the computational complexity of each query. It is rational to dedicate complex queries to nodes with higher resources. These nodes are usually in upper layers of hierarchical fog architecture. The window size of average query is equal to 50 in this evaluation.

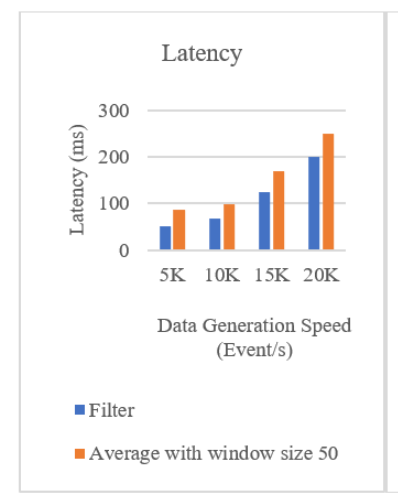

(a)

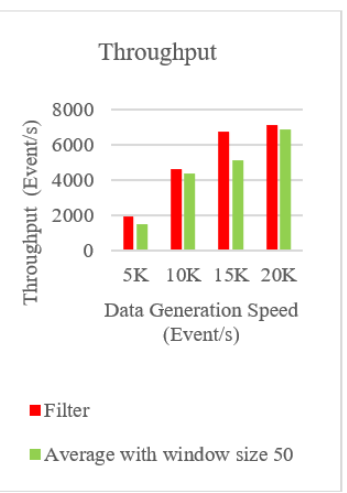

(b)
Fig. 11. Comparison between the filter and average query and the results for a) delay and b) throughput of WSO2 stream processor

\section{B. Analysis of resource utilization}

Running a container instance has three phases of processing, including container start-up, query-free execution, which is when the container is up without executing any query and query execution. Fig. 12 and Fig. 13 demonstrate the RAM and CPU usage of Apache Flink and WSO2 in the mentioned phases of processing. In both experiments, $2 \mathrm{~GB}$ of RAM and 2 CPU cores dedicated to each executed container. The Apache Flink container starts in 2 seconds (second \#0-\#2), and WSO2 container starts in 3 seconds (second \#0-\#3). For up to eight seconds, both containers continue to run without query. The second \#8 filter queries are assigned to both containers at stream data rates of 10,000 events per second. After second \#8, the average RAM consumption reached $683.2 \mathrm{MB}$ for Apache Flink and 661.88MB for WSO2. The average CPU consumption reached 12\% for Apache Flink and 10\% for WSO2.

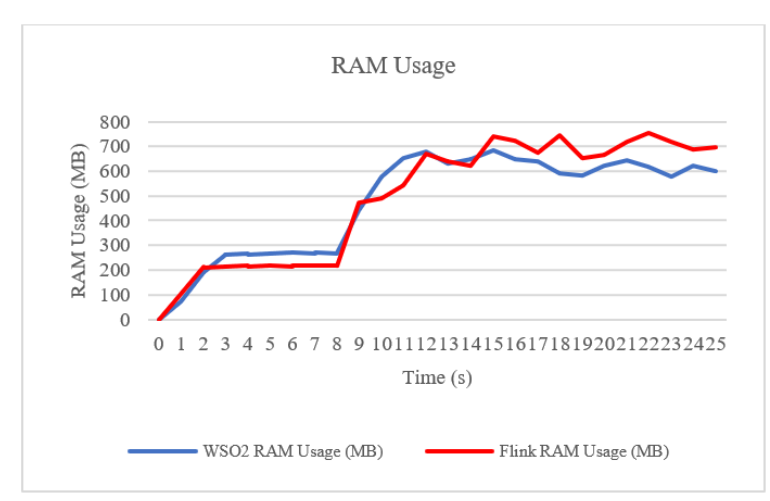

Fig. 12. WSO2 and Apache Flink RAM consumption

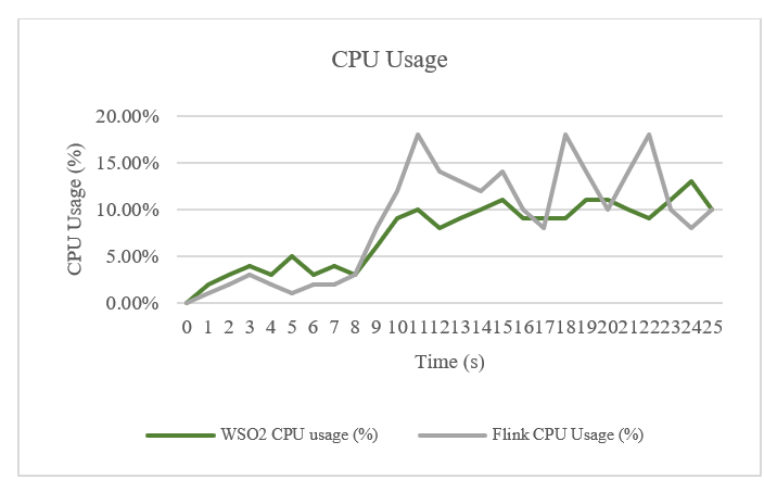

Fig. 13. WSO2 and Apache Flink CPU consumption

As a result, the containers used only about 33 percent of the allocated RAM and 11 percent of the allocated CPU for this query. They retained the remaining 67 percent of the allocated RAM and 89 percent of the allocated CPU unused. This residual value can be used for another query or application. Based on these experiments, all chromosomes with $A\left(Q_{\text {filter }}\right)=[\mathrm{w}, 2, \mathrm{z},>1024], A\left(Q_{\text {filter }}\right)=[\mathrm{x}, 1, \mathrm{y},>1024]$, $A\left(Q_{\text {filter }}\right)=[\mathrm{w}, 2,>0.5, \mathrm{z}]$, and $A\left(Q_{\text {filter }}\right)=[\mathrm{x}, 1,>0.5, \mathrm{y}]$ with an input rate of 10,000 events per second, can be excluded from offspring. 


\section{Analysis of GA generations}

This section evaluates the crucial factors influencing the number of generations required to achieve a placement to meet the application deadline. For example, the purpose of an application called autonomous driving requires processing to be finalized in 200 milliseconds. If the final processing time increases to more than 200 milliseconds, the application functionality may be disturbed.

1) Analysis of chromosome type: The number of available genes in chromosome type 3 is higher than type 2, and type 2 is greater than type 1 . Therefore, the number of states available for type 3 chromosomes will be higher than type 2 and type 1 . Fig. 14 illustrates the comparison of the number of generations required to achieve a hypothetical 200 milliseconds deadline in the three types of chromosomes.

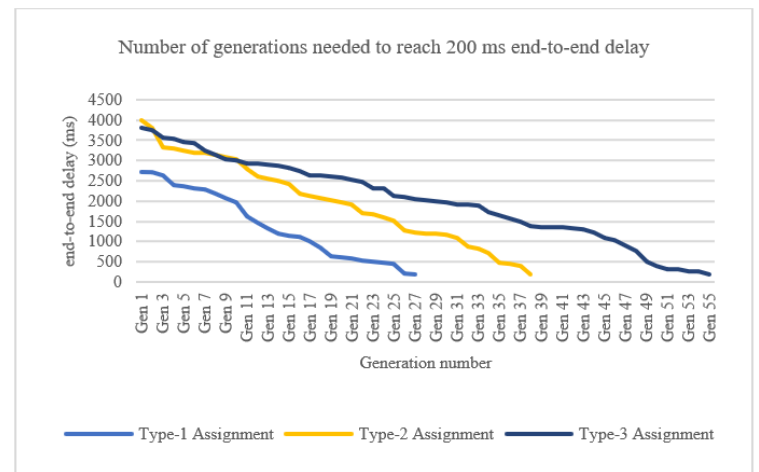

Fig. 14. Comparison of the number of generations required in the three types of chromosomes in a fog layer with 30 nodes and 5 queries. GA has been repeated 100 times.

Considering the higher number of chromosome type 3 genes than type 2 and type 1 , the results are reasonable. Type 3 chromosomes have four parts, and type 2 chromosomes have three distinct parts; each of them has to crossover and mutate separately to achieve the desired state. As a result, type 3 chromosomes require more generations to meet deadlines than type 2 and type 2 than type 1 . Since it takes time for crossover and mutation calculations to make the next generation, type 1 chromosomes are faster than type 2, and type 2 is quicker than type 3. So, it is faster to use chromosome type 1, but it should be considered that hardware resources will not be optimally utilized in chromosome type 1 due to its definition.

2) Analysis of the number of nodes and queries: In this section, we test the effect of the fog network's size and the number of application queries on the number of generations needed to reach a defined deadline. Fig. 15 compares the number of generations for two fog networks and two types of applications. These four modes have been tested for chromosome type 1 .

By doubling the number of queries on all three chromosome types, the number of genes per chromosome doubles. By doubling the number of fog nodes, the possible states for each gene increases. With the rise in the number of fog nodes and the number of queries, the average number of generations needed to reach the defined deadline increases.

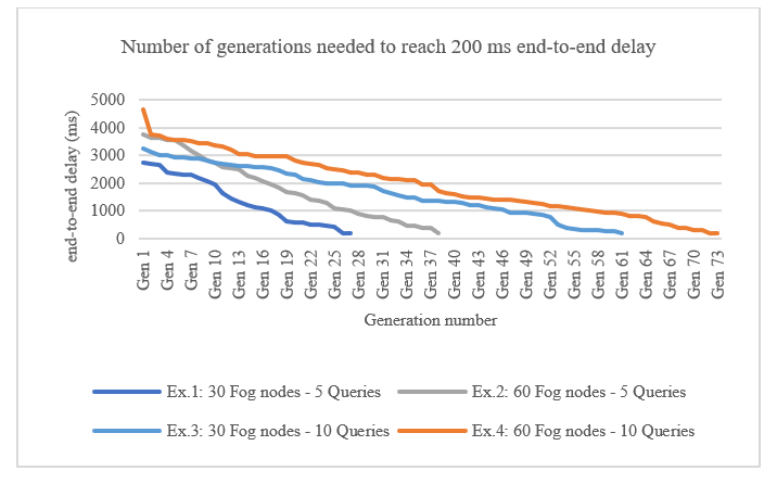

Fig. 15. Comparing the number of generations required in the four modes for the fog network and application queries, the GA has been repeated 100 times.

By considering individual offsprings and looking at the placement results, it can be concluded that by scheduling queries in the lower layers of fog, weak hardware resources can cause processing bottlenecks. In this case, the processing delay increases, but the network link delay decreases. By scheduling queries in the upper layers of fog, long-distance network links cause more network delay. In this case, the processing delay decreases, but the network link delay increases. Demonstrated experiments in Section IV-C2 shows that better (lower) fitness values provide better balance among nodes. Therefore, it becomes clear that the balanced assignment of queries among different fog layers would be the ideal allocation to minimize the end-to-end delay, provided node capabilities and link parameters are near or the same as a real-world scenario.

\section{COnClusions And Future Work}

This paper has proposed a general approach to query placement among the fog processing nodes to determine the low end-to-end delay value satisfying application deadlines. This solution applies to all SPEs, and researchers can use it to implement all real-world scenarios to find the near-optimal query placement. We are very first in emulation-simulation combination approach for stream processing queries allocation on fog layer that involves different types of SPEs and heterogeneous hardware resources.

As a future work, we can modify the crossover and mutation methods in the GA concerning the DAG structure of the application sequence to reduce the number of generations needed to meet the application deadlines. The paper's main idea can be expanded from the fog layer to the cloud-fog combination to address the workload balance between cloud and fog. In this paper, the stream processing parallelism level [27] in each node is 1 . We can consider other parallelism levels in future experiments to determine its effect on end-toend delay. Also, it is also possible to add power constraints to the fog nodes, in addition to the observed processor, memory, and communication link constraints. 


\section{REFERENCES}

[1] T. Pfandzelter and D. Bermbach, "IoT data processing in the fog: Functions, streams, or batch processing?," in Proceedings of IEEE International Conference on Fog Computing, pp. 201-206, 2019.

[2] S. Sagiroglu and D. Sinanc, "Big data: A review," in Proceedings of IEEE international conference on collaboration technologies and systems, pp. 42-47, 2013.

[3] K. Wähner, "Real-time stream grocessing as game changer in a big data world with hadoop and data warehouse," InfoQ, pp. 1-9, 2014.

[4] R. Casado and M. Younas, "Emerging trends and technologies in big data processing," Concurrency Computation, vol. 27, no. 8, pp. 20782091, 2015.

[5] S. Shahrivari, "Beyond batch processing: Towards real-time and streaming big data," Computers, vol. 3, no. 4, pp. 117-129, 2014.

[6] J. N. Hughes, M. D. Zimmerman, C. N. Eichelberger, and A. D. Fox, "A survey of techniques and open-source tools for processing streams of spatio-temporal events," in Proceedings of 7th ACM SIGSPATIAL International Workshop on GeoStreaming, pp. 1-4, 2016.

[7] S. Yang, "IoT stream processing and analytics in the Fog," IEEE Communications Magazine, vol. 55, no. 8, pp. 21-27, 2017.

[8] P. Varshney and Y. Simmhan, "Characterizing application scheduling on edge, fog, and cloud computing resources," Software - Practice and Experience, vol. 50, no. 5, pp. 558-595, 2020.

[9] V. T. N. Nguyen and R. Kirner, "Demand-based scheduling priorities for performance optimisation of stream programs on parallel platforms," in Lecture Notes in Computer Science, vol. 8285 LNCS, pp. 357-369, Springer, 2013.

[10] X. Liu and R. Buyya, "Resource management and scheduling in distributed stream processing systems: A taxonomy, review, and future directions," ACM Computing Surveys, vol. 53, no. 3, pp. 1-41, 2020.

[11] B. Peng, M. Hosseini, Z. Hong, R. Farivar, and R. Campbell, "R-storm: Resource-aware scheduling in storm," in Proceedings of 16th Annual Middleware Conference, pp. 149-161, 2015.

[12] P. Pietzuch, J. Ledlie, J. Shneidman, M. Roussopoulos, M. Welsh, and M. Seltzer, "Network-aware operator placement for stream-processing systems," in Proceedings of IEEE International Conference on Data Engineering, vol. 2006, p. 49, 2006.

[13] G. Amarasinghe, M. D. De Assuncao, A. Harwood, and S. Karunasekera, "A data stream processing optimisation framework for edge computing applications," in Proceedings of 21 st IEEE International Symposium on Real-Time Computing, pp. 91-98, 2018.

[14] V. Cardellini, V. Grassi, F. L. Presti, and M. Nardelli, "Optimal operator replication and placement for distributed stream processing systems," in Performance Evaluation Review, vol. 44, pp. 11-22, 2017.

[15] M. Dias de Assunção, A. da Silva Veith, and R. Buyya, "Distributed data stream processing and edge computing: A survey on resource elasticity and future directions," Journal of Network and Computer Applications, vol. 103, pp. 1-17, 2018.

[16] V. Cardellini, G. Mencagli, D. Talia, and M. Torquati, "New landscapes of the data stream processing in the era of fog computing," Future Generation Computer Systems, vol. 99, pp. 646-650, 2019.

[17] F. R. De Souza, M. Dias De Assuncao, E. Caron, and A. Da Silva Veith, "An optimal model for optimizing the placement and parallelism of data stream processing applications on cloud-edge computing," in Proceed ings of Symposium on Computer Architecture and High Performance Computing, vol. 2020-Septe, (Porto, Portugal), pp. 59-66, 92020.

[18] S. Vanneste, J. de Hoog, T. Huybrechts, S. Bosmans, R. Eyckerman, M. Sharif, S. Mercelis, and P. Hellinckx, "Distributed uniform streaming framework: An elastic fog computing platform for event stream processing and platform transparency," Future Internet, vol. 11, no. 7, 2019.

[19] M. Nardelli, V. Cardellini, V. Grassi, and F. Lo Presti, "Efficient operator placement for distributed data stream processing applications," IEEE Transactions on Parallel and Distributed Systems, vol. 30, no. 8, pp. 1753-1767, 2019.

[20] R. Ghosh and Y. Simmhan, "Distributed scheduling of event analytics across edge and cloud," ACM Transactions on Cyber-Physical Systems, vol. 2, no. 4, pp. 1-28, 2018.

[21] A. Brogi, G. Mencagli, D. Neri, J. Soldani, and M. Torquati, "Containerbased support for autonomic data stream processing through the fog," in Lecture Notes in Computer Science, vol. 10659 LNCS, pp. 17-28, Springer, 2018.

[22] T. Hiessl, V. Karagiannis, C. Hochreiner, S. Schulte, and M. Nardelli, "Optimal placement of stream processing operators in the fog," in Proceedings of 3rd IEEE International Conference on Fog and Edge Computing, pp. 1-10, 2019.
[23] R. Eidenbenz and T. Locher, "Task allocation for distributed stream processing," in Proceedings of IEEE International Conference on Computer Communications, vol. 2016-July, 2016

[24] H. J. Hong, P. H. Tsai, A. C. Cheng, M. Y. S. Uddin, N. Venkatasubramanian, and C. H. Hsu, "Supporting internet-of-things analytics in a fog computing platform," in Proceedings of the International Conference on Cloud Computing Technology and Science, 2017.

[25] M. T. Chung, N. Quang-Hung, M. T. Nguyen, and N. Thoai, "Using docker in high performance computing applications," in Proceedings of 6th IEEE International Conference on Communications and Electronics, pp. 52-57, 2016.

[26] V. Chandel, S. Patial, and S. Guleria, "Comparative study of testing tools blazemeter and apache jmeter," Sukkur IBA Journal of Computing and Mathematical Sciences, vol. 2, no. 1, pp. 70-76, 2018.

[27] H. Röger and R. Mayer, "A comprehensive survey on parallelization and elasticity in stream processing," ACM Computing Surveys, 2019.

Hamed Hasibi received the bachelor and M.S. degrees in computer science from K. N. Toosi University of Technology, Tehran, Iran. His research interests include Distributed Computing, Bioinformatics, and Artificial Intelligence.

Saeed Sedighian Kashi received his B.Sc. (2003), M.Sc. (2005), and Ph.D. (2012) in Computer Engineering from Iran University of Science and Technology, Tehran, Iran. Currently, he is an assistant professor in K. N. Toosi University of Technology, Tehran, Iran. His research interests include Wireless Sensor Networks (WSNs), Internet of Things (IoT), Service-Oriented Architecture (SOA), and Cloud Computing. 